\title{
Metabolism, Transport, and Morphogenesis: Which Drives Which?
}

\author{
BY P. MITCHELL \\ Chemical Biology Unit, Department of Zoology, University of Edinburgh
}

In the course of the last forty or fifty years, the profound insight that biochemists have obtained into the biological processes known collectively as energy metabolism has led to the belief amongst many biochemists and cell biologists that the processes of transport, growth, and morphogenesis are 'driven' by the 'energy released through metabolism'. This popular belief has, to some extent, substituted the imaginary demon of 'metabolic energy' for the vis vitae. In the case of 'active transport' across membranes, for example, many of us tend to visualize a kind of grabbing or catapult system that is triggered off by contact with its substrate so that it traps the substrate and, with the aid of 'metabolic energy', forces the substrate through the membrane, where it releases it and passes back again in readiness to grab another. With morphogenesis it is somewhat harder to portray how we may imagine the process to be 'driven' by metabolic energy, but I am sure that our mental images often tend to include miniature conveyor belts, substrate-specific mechanical shovels, and all kinds of engineering devices. As Waddington (1959) has pointed out in an excellent little cameo on physico-chemical aspects of morphogenesis, 'It is perhaps remarkable that biology, which was for so long a morphological science, has made so little attempt, now that it has passed into the experimental phase, at a causal analysis of the mechanisms that give rise to the shapes which have been so extensively studied'. Waddington was mainly thinking of multicellular organisms, but although the microbiologist has the great advantage of studying morphogenesis in individual cells or in quite small aggregates, Waddington's conclusion about our ignorance of the morphogenetic mechanisms is nevertheless largely true.

Of course, the fundamental advances in the knowledge of the molecular mechanisms of transport, growth and morphogenesis have had to await the development of their foundations in classical biochemistry. But $I$ agree with Waddington and with other biologists who are suggesting, in effect, that it would now be helpful if the biochemically minded were more often to interrupt their preoccupation with the chemical foundations, to straighten their backs, as it were, and to look carefully at the master plans of our science-that is, at whole living organisms.

When we consider whole adapting and developing bacteria and give our thoughts some licence within the context of the present symposium, we may realize that the processes of growth and morphogenesis represent the movements of chemical groups (atomic nuclei and electrons) at certain rates and in certain definite directions in space, from nutrients initially present in the growth medium to polymers or metabolic intermediates (including inorganic ions and water) within the organism. Thus, growth and morphogenesis represent transport processes which, in common with the more popular membrane transport, must be described by vector quantities, having both magnitude and direction in space. They can be represented by arrows, 
the length of which will denote their magnitude. On the other hand, orthodox chemical reactions are scalar quantities, having only magnitude and not direction in space. They can be represented by dots of different size, or by numbers. It is a matter of experience-and of theory (see Curie, 1908) - that scalar or directionless quantities cannot determine the direction of vector quantities. And so, the spatially directionless processes of metabolism (as usually understood) cannot force the movement of any chemical against the natural direction of diffusion (see Jardetsky $\&$ Snell, 1960; Kedem, 1961). This is equivalent to saying that if a substance appears to be moving against the natural diffusion gradient in a resting or growing organism (that is, if it seems to be 'actively' transported) the substance in question cannot be doing so, but must either be moving as some unidentified derivative, or must be carried by a bulk flow (Mitchell, 1959 $b, 1961 a, b$ ). Pausing for a moment, you will see that the simple argument that we have just used implies that the cause of the process usually described as 'active transport' must be nothing more than the thermal or Brownian motion that gives rise to down-hill diffusion of molecular complexes, molecules, or stable ions, or the pressure gradient that gives rise to the down-hill movement of a mass of fluid (or of solid fibrils or particles), and which must be due, in the first place, to a diffusion or escaping process.

Apart from its intrinsic interest, this conclusion is very valuable to the experimentalist, for it may impel him not to rest content with the sort of 'active transport' model that is based upon macroscopic mechanical analogies, but to ask what the unidentified derivative of the apparently 'actively transported' substance may be, or whether the transport may be due to a bulk flow. One may object that this view of 'active transport' as a diffusion or down-hill escape, robs the idea of its glamour and mystery; and, for example, we would have to discard the romantic suggestion of Cohen \& Monod (1957) that membrane transport is controlled by demons like those imagined (but not isolated and purified!) by Clerk Maxwell. I think, however, that we have to accept this possible loss of romance as one of the normal consequences of scientific thought and enlightenment; and there are indeed signs that the Parisian permease school are beginning to recognize this necessity (see Kepes, 1960). So far our argument has suggested that we can represent the process of membrane transport and the vectorial processes involved in the activities of maintenance and growth by processes of diffusion alternating with chemical transformation. The chemical transformation (promoted by an enzyme) gives rise to the disappearance of a given component and the appearance of another at a certain site so that diffusion of the appropriate components towards and away from the site of chemical change will occur. This description, as you see, seems to distinguish sharply between chemical transformation and diffusion.

During the last few years it has become apparent that certain types of catalysed chemical process (involving the exchange of covalent bonds) are not strictly scalar. These are what I have called group-translocation reactions, because the chemical process of group transfer is accompanied by the effective diffusion of chemical groups across an anisotropic catalytic system (Mitchell, 1957, 1959 $a, b$, 1961 $a, b, c$; Mitchell \& Moyle, 1958 $a, b)$.

Figure 1 illustrates a working model of a hydroxyl-translocating ATPase as an example of a catalyst of group translocation. This model helps one to realize that although we are accustomed to thinking of the diffusion and the chemical change 
of molecules in rather different terms, the processes involved in diffusion and chemical change are, in fact, very similar. The diffusion of a molecule or ion or molecular complex in a biological system describes the movement of the particle by the thermally activated breaking and making of the secondary bonds that tend to prevent the displacement of the particle relative to the neighbouring atoms. The chemical

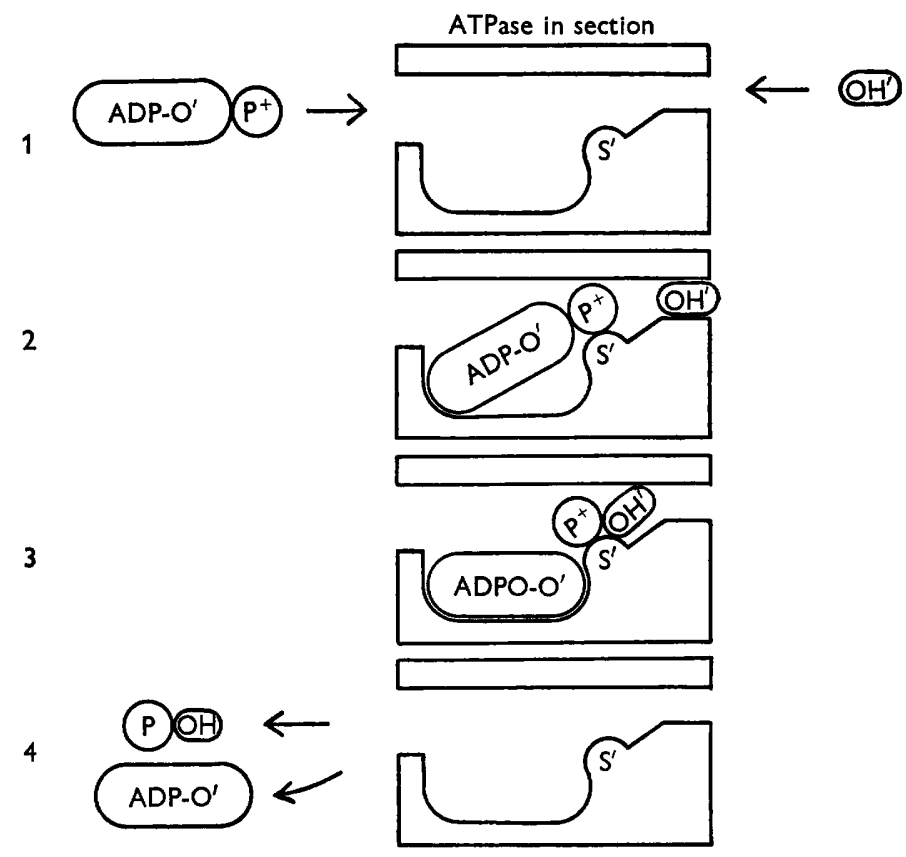

Fig. 1. Diagram of working model of anisotropic ATPase, translocating hydroxyl ion. Reaction shown in stages 1 to 4. The channel leading to the active centre from the left allows passage of adenosine triphosphate (ADP-O $\left.-\mathrm{P}^{+}\right)$, phosphate $(\mathrm{POH})$ and $\mathbf{H}^{+}$, but not of $\mathrm{OH}^{\prime}$. The right-hand channel allows passage of $\mathrm{OH}^{\prime}$ only. The phosphorylium group (represented as $\mathbf{P}+$ - equivalent to monomeric metaphosphate) forms an intermediate with a negatively charged atom $\left(S^{\prime}\right)$ in the enzyme.

transformation of a molecule or ion or molecular complex describes the movement of one of its constituent chemical groups by the thermally activated breaking and making, not only of secondary bonds, but also the primary bond that tends to prevent the escape of the group from its partner (or donor group) and its transfer to an acceptor group.

If, then, we think of chemical transformation as being due to the diffusion of chemical groups (and/or electrons) from group donors to group acceptors, guided or catalysed at certain points by the presence of group-transferring and grouptranslocating enzymes, it is evident that we will have to regard the gradient of escaping tendency, or the gradient of 'group potential'-as Lipmann (1941, 1960) called it-not only as the determinant of the direction of chemical reaction in the orthodox scalar sense, but also in the vector sense, meaning the direction of group diffusion or transport.

We have arrived now at a more homogeneous view of the processes of diffusion 
and chemical reaction in which the first refers to the down-hill escape of molecular complexes, molecules or stable ions, and the second refers to the down-hill escape of chemical groups, electrons, or radicals. As recognized by Henry Eyring and others (see Glasstone, Laidler \& Eyring, 1941), the processes of diffusional and chemical change are alike in being activated diffusion processes. Further, in condensed systems such as we mainly find in biology, these thermodynamic processes proceed by a partially reversible random bouncing of the particles from less probable configurations to more probable configurations in space. The activity of enzymes and catalytic carriers in such systems is due to their capacity for increasing the probability of certain transitional spatial configurations. This is the justification for thinking of metabolism as a spatially organized process of translocation catalysis (Mitchell, 1959a, $b, 1961 a, b, c$ ).

It is now necessary to return to our argument about the impossibility of driving substances against the natural diffusion or escaping tendency by chemical reactions, for in the case of the group-translocation type of reaction, the chemical process as we have now defined it contains a spatially directed or vectorial element. In the case of the model of the ATPase that translocates hydroxyl ions, for example, we can see that the movement of hydroxyl across the molecule is dependent upon the electrochemical potential gradient of hydroxyl ion across the system-bearing in mind, of course, that the effective hydroxyl ion activity on the left is not that of the free hydroxyl ion, but is the activity of the $\mathbf{O H}^{\prime}$ group in inorganic phosphate.

Now, it is clearly possible that the vectorial transfer of hydroxyl ions through the anisotropic ATPase might influence the movement of other molecules or ions (say $\mathrm{Na}^{+}$or $\mathrm{K}^{+}$) along with it by a momentum transfer process. But it is sufficient to note for the purposes of the present argument that even if this were the case, the direction and velocity of the group translocation reaction would still depend upon the diffusion gradient of the effective hydroxyl ion-alkali metal ion 'complex' across the catalytic system as a whole. The particles involved in translocation cannot as a group go against the direction of the natural escaping tendency.

To summarize, it seems that in the metabolic sequence (that we described earlier as diffusion alternating with chemical transformation) no step can properly be said to drive the processes of transport or metabolism forward, for every step in reality represents a tendency for molecules or chemical groups to escape from one place and pass to another, spontaneously.

\section{THE CYTOSKELETON}

One may well ask how the processes of vectorial metabolism that I am systematically formulating in this paper come to be organized in space, in the macroscopic sense. We will now go on to consider this question very briefly.

When the enzymes, catalytic carriers, and other regulators of diffusion in a system catalysing a metabolic process are distributed homogeneously or randomly, there will, of course, be no macroscopic spatial consequence of the movements of the substrates relative to the catalysts; for such movements, being directed at random, will have no vector component. This is metabolism as we often see it in the test tube. On the other hand, if the microscopically anisotropic catalysts are organized 
in space upon or within a macroscopically anisotropic membrane or other structure, the corresponding spatially organized diffusion of the substrate molecules and chemical groups in the system represents the fundamental vectorial component of the metabolic process underlying 'active' transport and developmental processes generally.

The anisotropic array of catalysts required to produce the spatial and metabolic organization of the organism must, of course, be supported upon or within a specifically locating framework (Mitchell, $1959 b, 1961 a, b$ ). Thus we have formulated, in explicit biochemical terms, the requirement for the cytoskeleton envisaged long ago, for example by Peters and by Needham (see Peters, 1939). In bacteria we are lucky in being able to recognize the cell-wall and the plasma membrane as the main cytoskeletal components-the backbone, as it were, of the whole spatial metabolic organization (see Mitchell \& Moyle, 1956a).

I hope it will now be possible to see more clearly the practical aim of this rather abstract exposition. My aim has been to draw your attention to the spatial asymmetry of the individual catalysts and the spatial asymmetry of the organized polymolecular systems in which they reside. For this spatial asymmetry can properly be regarded as a primary cause (or specification) of the vectorial diffusion processes of organized metabolism and growth.

It seems rather unlikely that during the growth of micro-organisms many of the enzymes and catalytic carriers of the cell envelopes and cytoplasm could be synthesized at their sites of activity, for the necessary synthetic machinery could hardly be fitted into the available space; and indeed, there is evidence, which will be discussed in this symposium, that the proteins (or at least their subunits) are made in the microsomal particles. We must therefore consider the possibility that the very catalytic proteins that are to cause and control the translocation of specific substrates across the plasma membrane, or the translocation and incorporation of specific chemical groups into cell-wall polymers, and so on, must themselves possess the specificities that will allow them to diffuse to and become specifically bonded into their sites of activity in the polymolecular fabric of the cell envelopes and cytoplasm during growth and adaptation (Mitchell, 1959b, $c, 1961 b$ ). Thus, we are given a new reason for investigating the idea, originally suggested by Green (1957), that enzymes and catalytic carriers have a dual specificity-one being the substrate specificity, and the other being a locational specificity dependent on the shape and properties of the surface of the protein. According to this conception the locational bonds would represent the articulations between the 'small bones' of the cytoskeleton.

It is against this kind of background that the studies of the distribution of enzyme activities between the plasma membrane and cytoplasm fractions of microorganisms are of real biological interest. I do not propose to dwell upon distribution studies; Dr Dawson will be dealing with them in some detail. But I think it would be appropriate to mention just a few germane facts. Some time ago, Keilin \& King (1960) showed that after the succinic dehydrogenase protein of the cytochrome complex of the mitochondrial membrane fraction has been displaced (or 'solubilized') by changing the physical conditions of the system, it will, under normal physiological conditions, pass back into fresh succinic dehydrogenase-poor cytochrome complex, becoming 'insoluble' and functional again as part of the succinic oxidase system. 
Dr Moyle and I have succeeded in showing a similar reversibility of the binding of $\alpha$-ketoglutarate dehydrogenase in the membrane complex of Micrococcus lysodeikticus (see Mitchell, 1961b). Very recently, Green and his collaborators (Green, Tisdale, Criddle \& Bock, 1961) have isolated a protein of low water solubility from mitochrondria which may represent an important structural element in the membrane, and they have obtained some evidence that this protein forms complexes with certain of the enzymes of the electron and hydrogen transport system. Miss Thomson and I have found that about $20 \%$ of the total nitrogen of the plasmamembrane material of $M$. lysodeikticus is soluble in chloroform + methanol $(2+1, v / v)$, and that practically all this nitrogen is accounted for by a protein of low water solubility, the amino acid analysis of which shows an unusually high proportion of non-polar amino acids. The surface-active properties of this protein suggest that it is an important structural element of the plasma membrane. The studies of protein synthesis that Dr McQuillen, Dr Dagley, and Dr Hunter are to describe at this symposium and the ideas on cell-wall synthesis that Dr Salton has already discussed presumably require spatially anisotropic molecular complexes in microsome and plasma membrane, and the experimental work on the reversible dissociation of these supramolecular complexes is beginning to support a jig-saw puzzle view of much of the cytoskeleton, many of the bits of the jig-saw staying together mainly because they fit. One would say, in more strictly chemical terminology, that the molecules of the cytoplasmic and membrane complexes are probably mostly linked together by residual valencies and by hydrophilic bonding, as, for example, in the case of the four subunits of haemoglobin (see Kendrew, 1961).

Now I would like to return to the main theme of my paper and describe very briefly some experiments that we have recently done in Edinburgh in an effort to demonstrate the anisotropy of certain enzyme systems in the plasma membrane of bacteria.

\section{VECTORIAL METABOLISM}

Many of the current ideas about the molecular mechanism of metabolically driven ion-transport begin with the assumption that the components of the metabolic electron and hydrogen transport system are arranged in a spatial sequence across the membrane through which transport occurs. Lundegardh (1945) was the originator of this idea, which we can represent as shown in Fig. 2. In terms of enzymology, this might imply that cytochrome oxidase (or other terminal catalyst for donating electrons to oxygen) would be situated on the right, and that DPNH dehydrogenase and succinic dehydrogenase, for example, would be at the left-hand side; so that the passage of electrons through the membrane (perhaps via the cytochrome $\mathrm{Fe}^{2+} /$ $\mathrm{Fe}^{3+}$ system) would form $\mathrm{OH}^{\prime}$ ions on the right and $\mathrm{H}^{+}$ions on the left in equal numbers.

Since biochemists have often considered some such spatial arrangement of the cytochrome system and associated enzymes across mitochondrial and other membranes, and have assumed that it might be of physiological importance (see Robertson, 1960), I thought it would be useful to attempt to test the possible asymmetry of the oxidation-reduction processes across the plasma membrane of Micrococcus lysodeikticus by a suitable combination of metabolic and transport studies. But, before I talk about this work, it will be necessary to give you some background facts. 
Some years ago, Dr Moyle and I found that the bulk of the cytochrome system of Micrococcus lysodeikticus is present in the plasma membrane of the cells. We also found that the plasma membrane contains several oxidative enzymes, including succinic oxidase. At $\mathrm{pH} 7$, the plasma membrane of $M$. lysodeikticus is impermeable to hydrophilic solutes such as ATP, DPN, glucose, succinate, malonate, and ferricyanide (Mitchell \& Moyle, 1956 $b$, and unpublished observations). On the other hand, it is freely permeable to lipid-soluble solutes such as ethanol. Recently I have confirmed the observation of Gilbey \& Few (1958) that $\mathrm{H}^{+}$ions pass only slowly through the plasma membrane of $M$. lysodeikticus, and have made the surprisingly elementary discovery that uncouplers of oxidative phosphorylation and assimilation, such as 2:4-dinitrophenol, salicylate, dicoumarol, and azide, catalyse the equilibration of $\mathrm{H}^{+}$ions in proportion to their uncoupling activity (Mitchell, $1961 d)$.

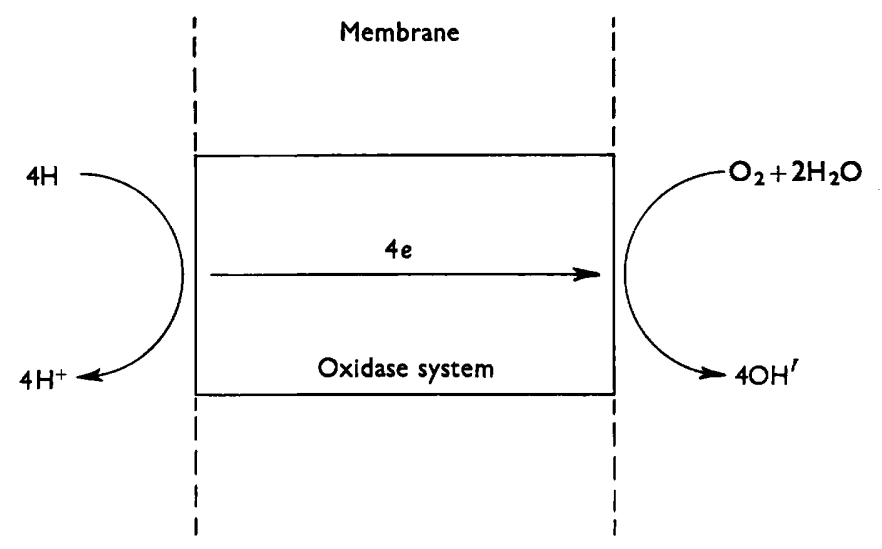

Fig. 2. Diagram of anisotropic oxido-reduction system after Lundegardh (1945).

With these facts as a background, let us focus attention upon the succinic oxidase system in the membrane of Micrococcus lysodeikticus. The intact cells oxidize externally added succinate rapidly, using molecular oxygen-but this oxidation is not depressed by the presence of malonate outside the cells. (Malonate, of course, is the classical competitive inhibitor of succinic dehydrogenase.) If, however, the $\mathrm{pH}$ of the cell suspension is briefly depressed to 5 so as to let in externally added malonate without otherwise damaging the cells, the oxidation of succinate is almost completely abolished. These observations demonstrate that the succinate and malonate accepting region of the succinic oxidase system of this organism is situated at the inner side of the plasma membrane as shown in Fig. 3. I should, perhaps, remind you that the succinate cannot pass spontaneously through the membrane as such, but goes via a highly specific catalytic system, probably as succinyl, which we will have to take for granted for the purpose of the present argument.

The second fundamental question, concerning the possible asymmetry of the succinic oxidase system that one would naturally ask is, where, in relation to the plasma membrane, is the cytochrome oxidase? A few years ago, Chance $(1953 a, b)$ described a carbon monoxide spectrum of the cytochrome oxidase of this organism, and so we carried out experiments designed to locate this terminal oxidase. To our 
surprise we discovered, in the course of these studies, that the respiration of Micrococcus lysodeikticus is not depressed by $10^{-4} \mathrm{M}$-cyanide or $10^{-2} \mathrm{M}$-azide-indeed, it is stimulated by 30-50\%-and so we were forced to the conclusion that this highly aerobic organism does not contain a cytochrome oxidase of normal cyanide or azide sensitivity. This being the case, we had to abandon our plan to locate the

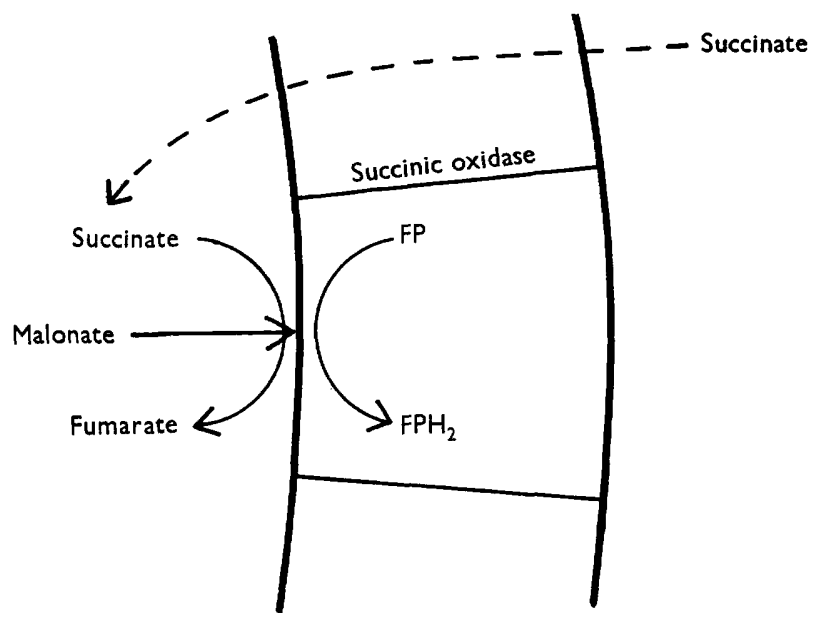

Fig. 3. Arrangement of succinic dehydrogenase flavoprotein (FP) relative to the plasma membrane of Micrococcus lysodeikticus. Malonate can inhibit only from inside.

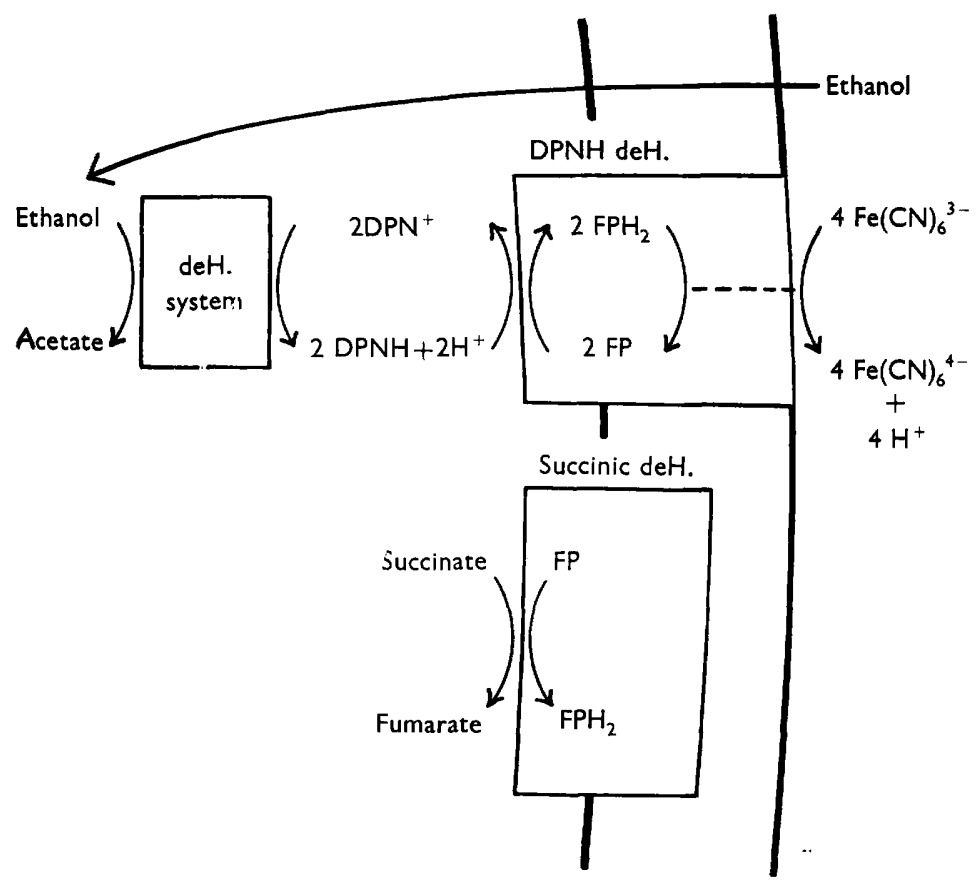

Fig. 4. Suggested arrangement of dehydrogenase systems in relation to reduction of external ferricyanide, forming hydrogen ions outside the plasma membrane of Micrococcus lysodeikticus. The dotted line may represent a quinol/quinone system. 
oxidase with non-penetrating inhibitors. Instead, we decided to search for an outlet of electrons on the external surface of the plasma membrane by using the non-penetrating ferricyanide ion as electron acceptor in place of oxygen, and to look for a possible inhibitory action of ferricyanide on oxygen uptake. We observed that, although external ferricyanide could not substitute for or inhibit molecular oxygen uptake in the oxidation of succinate by suspensions of intact cells, it could accept electrons arising from the oxidation of ethyl alcohol to acetic acid via a DPN-linked dehydrogenase system. Further, alcohol oxidation was carried out at

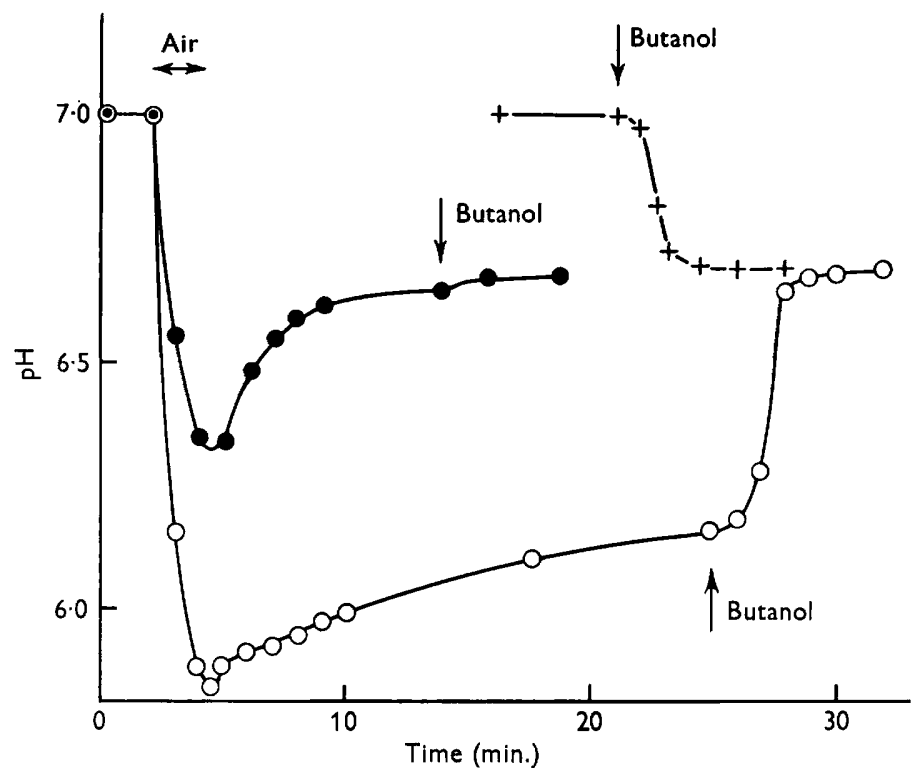

Fig. 5. Change of $\mathrm{pH}$ of suspension medium $(0 \cdot 1 \mathrm{M}-\mathrm{KCl})$ by suspensions of Micrococcus lysodeikticus (10 mg. dry wt./ml.). The washed suspensions (which exhibit vigorous endogenous respiration) are initially adjusted to $\mathrm{pH} 7$ anaerobically, bubbling with pure $\mathrm{N}_{2}$. Lower curve (-O-O- ), suspensions aerated for $2 \mathrm{~min}$. and then immediately made anaerobic again. Butanol added $(5 \%, v / v)$ at arrow. Similar experiment in presence of $10^{-3} \mathrm{M}-2: 4$-dinitrophenol (- - - Experiment omitting aeration, and without addition of dinitrophenol $(-+-+-)$.

the same very rapid rate by ferricyanide as by molecular oxygen; and the hydrogen ions produced in the course of the reduction of ferricyanide were found by titration in unbuffered incubation media to be liberated exclusively outside the membrane of the cells. Since externally added DPN could be shown not to participate in the DPN-linked dehydrogenase systems of intact cells, and since the normal DPNlinked reactions ceased if the cell membrane were damaged so as to allow the endogenous DPN to escape, we concluded that, using ferricyanide as terminal electron acceptor, the oxido-reduction systems are probably arranged in some such way as illustrated in Fig. 4.

We were particularly impressed by the fact that, using ferricyanide as terminal electron acceptor, the hydrogen ions produced by the oxidation of alcohol were all released outside the membrane. This led us to inquire whether a similar production of external acid occurred when oxygen was used as terminal electron acceptor; for 
it would be expected that, since the reduction of oxygen can be equivalent to the production of hydroxyl ions, the hydrogen ions would be neutralized by hydroxyls if the site of the oxygen-terminal oxidase were outside the membrane, while they would not be neutralized if the oxygen-terminal oxidase were inside the membrane. We assumed, of course, that the oxygen, unlike the ferricyanide, can pass readily through the membrane. 'To try to locate the production of $\mathrm{OH}^{\prime}$ ions from the molecular oxygen, we measured the effect of a brief period of aeration on the external $\mathrm{pH}$ of

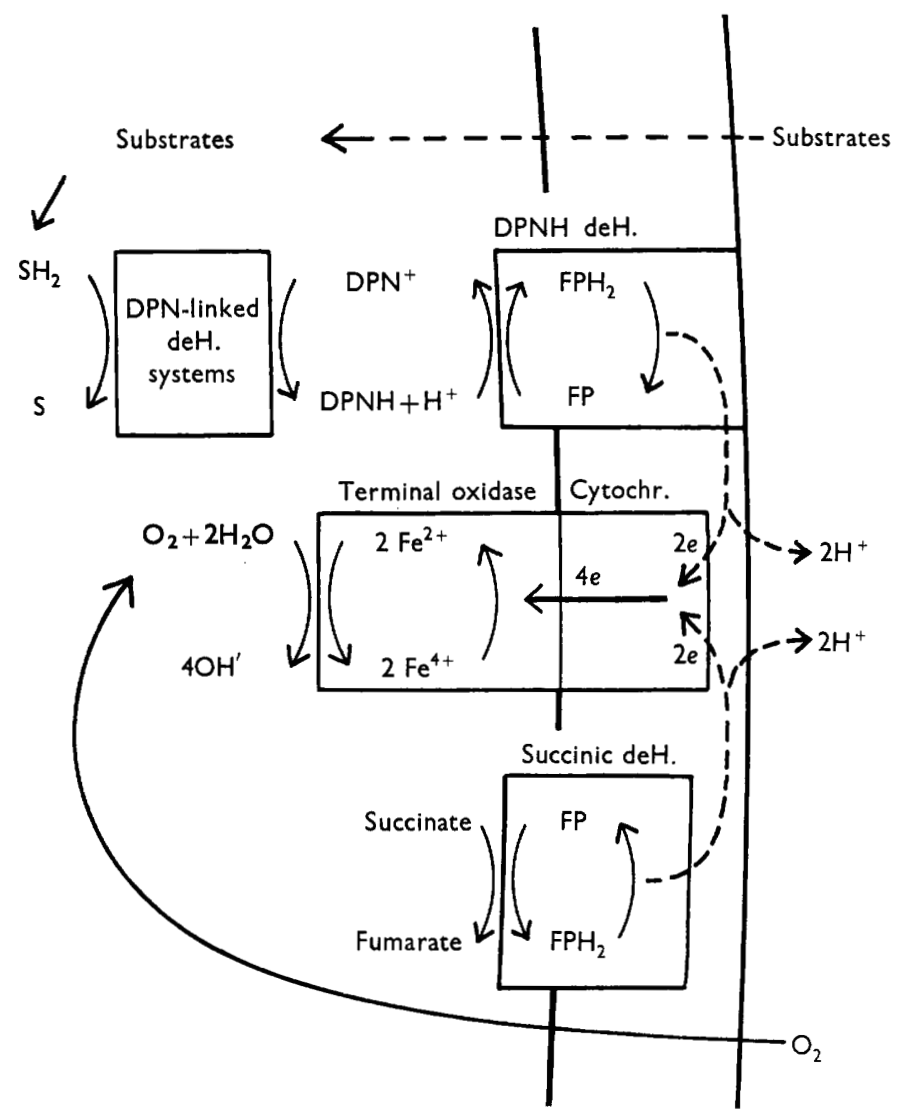

Fig. 6. Suggested arrangement of dehydrogenase systems to account for shift of 'acid' across the plasma membrane of Micrococcus lysodeikticus on aeration. Oxidizable substrates in cell are represented as $\mathbf{S H}_{2}$, and flavoprotein as FP. The dotted lines may represent quinol/quinone systems. The main feature of the system is that hydrogen atoms pass to outer surface of membrane, where they give up electrons that flow back, down to oxygen on the inside. It is like the system suggested by Lundegardh (1945), but bent back on itself.

unbuffered suspensions of Micrococcus lysodeikticus containing endogenous substrates. Figure 5 shows a typical group of observations. In normal cell suspensions, the admission of air causes a rapid depression of the $\mathrm{pH}$ of the suspension medium, and when, two minutes later, anaerobic conditions are reimposed by bubbling with pure nitrogen, the $\mathrm{pH}$ rises somewhat and then comes almost to rest about $0 \cdot 8$ units 
lower than it started. The addition of $n$-butanol at this point to give a final concentration of $5 \%(\mathrm{v} / \mathrm{v})$ allows the $\mathrm{pH}$ to rise to approximately $6 \cdot 6$ - the same $\mathrm{pH}$ as that obtained if butanol is added at the beginning of the experiment, no air having been admitted. As, on breaking the plasma membrane with $n$-butanol, the same final $\mathrm{pH}$ was attained whether the cell suspension had been aerated for $2 \mathrm{~min}$. or not, it was evident that the depression of the $\mathrm{pH}$ of the suspension medium could not be due to net acid production, but must be caused by the development of an asymmetry of the electrochemical potential of $\mathrm{H}^{+}$across the membrane. This was confirmed by the addition of 2:4-dinitrophenol at the beginning of the aeration experiment, for, as would be expected, the dinitrophenol decreased the asymmetry without affecting the final equilibrium pH. It was significant that in the normal cell suspensions, the initial rate of effective proton transfer during aeration corresponded to approximately $50 \mu$ equiv. $\mathrm{H}^{+} / \mathrm{g}$. cell dry wt. min., representing the release of between one and two protons outside the membrane (and an equivalent number of hydroxyl ions inside) per oxygen atom consumed. Figure 6 shows a tentative scheme to account for these observations. Experiments now in progress are providing similar evidence in the case of Escherichia coli (strain ML30).

\section{CONCLUSIONS}

Let me conclude my paper by asking: why is the oxido-reduction system anisotropic, causing the molecular oxygen and substrates that diffuse from the medium to liberate $\mathrm{OH}^{\prime}$ ions in the cytoplasm and $\mathrm{H}^{+}$ions outside? I believe the answer to be that this primary type of anisotropy has evolved because it causes, by processes of exchange, other secondary asymmetries that underlie the activities of assimilation and growth. One directed or vectorial chemical process tends to cause a primary asymmetry that may, in turn, lead to other secondary vectorial or transport processes and corresponding asymmetries. The oxido-reduction asymmetry is probably the cause of the membrane potential, it may well cause the asymmetry of distribution of $\mathrm{Na}^{+}$and $\mathrm{K}^{+}$, and of other substrates (such as sugars and amino acids) across the membrane; and it may, as I have recently suggested (Mitchell, 1961 c) give rise to oxidative phosphorylation by allowing a hydroxyl-translocating ATPase reaction (Fig. 1) to run backwards across the membrane. I would like to mention, in passing, that Dr R. E. Davies has drawn my attention to the fact that a suggestion, formally akin to the latter, was tentatively put forward some ten years ago by Davies \& Ogston (1950) and by Davies \& Krebs (1952).

We must not lose sight of the fact that transport processes in biology are integral with the activities of growth and survival. When we separate transport from morphogenesis, growth, and movement in living organisms, the separation is not real, but abstract. Thus, it emerges that the activities of biological transport represent the elusive directiveness of the phenomena of life. Scalar (or directionless) biochemistry is a subject of dead things, like crystals. To describe the flame-like properties of living things, we have to represent the metabolic processes as projections in space as well as in time. This requires the recognition and development of a new subject: the subject of vectorial chemistry. Perhaps, once again, the exact sciences are to receive inspiration from biology! 
I am indebted to many colleagues, and especially to Dr Jennifer Moyle, for helpful discussion during the preparation of this paper. It is also a pleasure to acknowledge the receipt of grants from the Nuffield Foundation in aid of this work.

\section{REFERENCES}

Chance, B. (1953a). The carbon monoxide compounds of the cytochrome oxidases. I. Difference spectra. J. biol. Chem. 202, 383.

Chance, B. $(1953 b)$. The carbon monoxide compounds of the cytochrome oxidases. II. Photodissociation spectra. J. biol. Chem. 202, 397.

Cohen, G. N. \& Monod, J. (1957). Bacterial permeases. Bact. Rev. 21, 169.

CurIE, P. (1908). Oeuvres, p. 127. Paris: Gauthier-Villars.

Davies, R. E. \& Krebs, H. A. (1952). Biochemical aspects of the transport of ions by nervous tissue. Biochem. Soc. Symp. 8, 77 .

Davies, R. E. \& Ogston, A. G. (1950). On the mechanism of secretion of ions by gastric mucosa and by other tissues. Biochem. J. 46, 324.

Gilbey, A. R. \& Few, A. V. (1958). The permeability of Micrococcus lysodeikticus to hydrochloric acid. Biochim. biophys. Acta, 30, 421.

Glasstone, S., Laidler, K. J. \& EYring, H. (1941). The Theory of Rate Processes. New York: McGraw-Hill.

Green, D. E. (1957). Organisation in relation to enzymic function. Symp. Soc. exp. Biol. $10,30$.

Green, D. E., Tisdale, H. D., Criddle, R. S. \& Bock, R. M. (1961). The structural protein and mitochondrial organisation. Biochem. Biophys. Res. Comm. 5, 81.

JARDETSKY, O. \& SNELl, F. M. (1960). Theoretical analysis of transport processes in living systems. Proc. nat. Acad. Sci., Wash. 46, 616.

KeDEM, O. (1961). Criteria of active transport. In Membrane Transport and Metabolism, p. 87. Ed. A. Kleinzeller \& A. Kotyk. Prague: Publ. House of Czechoslovak Acad. Sci.

KeIIIN, D. \& KING, T. E. (1960). Effect of inhibitors on the activity of soluble succinic dehydrogenase and on the reconstitution of the succinic dehydrogenase-cytochrome system from its components. Proc. Roy. Soc. B, 152, 163.

KENDREw, J. C. (1961). The structure of globular proteins. In Biological Structure and Function, Vol. 1, p. 5. Ed. by T. W. Goodwin \& O. Lindberg. London: Academic Press.

KePEs, A. (1960). Études cinétiques sur la galactoside-permease d'Escherichia coli. Biochim. biophys. Acta, 40, 70.

LipManN, F. (1941). Metabolic generation and utilization of phosphate bond energy. Advanc. Enzymol. 1, 99.

LipmanN, F. (1960). Attempts towards a formulation of biological use of energy in terms of chemical potentials. In Molecular Biology, p. 37. Ed. D. Nachmansohn. New York: Academic Press.

LUNDEGARDH, H. (1945). Absorption, transport and exudation of inorganic ions by the roots. Arkiv Bot. 32 A, 12, 1.

Mrtchell, P. (1957). A general theory of membrane transport from studies of bacteria. Nature, Lond. 180, 134.

Mitchell, P. (1959a). Structure and function in microorganisms. Biochem. Soc. Symp. 16, 73.

Mitchell, P. (1959b). Biochemical cytology of microorganisms. Annu. Rev. Microbiol. $13,407$.

Mrtchell, P. (1959c). In discussion. Proc. Roy. Phys. Soc. (Edinb.), 28, 77.

Mitchell, P. (1961 $a)$. Biological transport phenomena and the spatially anisotropic characteristics of enzyme systems causing a vector component of metabolism. In Membrane Transport and Metabolism, p. 22. Ed. A. Kleinzeller \& A. Kotyk. Prague: Publ. House of Czechoslovak Acad. Sci.

Mrtchen, P. (1961b). Approaches to the analysis of specific membrane transport. In Biological Structure and Function, Vol. 2, p. 581. Ed. by T. W. Goodwin \& O. Lindberg. London: Academic Press. 
MrTCheld, P. (1961 c). Coupling of phosphorylation to electron and hydrogen transfer by a chemi-osmotic type of mechanism. Nature, Lond. 191, 144.

Mitchell, P. (1961 d). Conduction of protons through the membranes of mitochondria and bacteria by uncouplers of oxidative phosphorylation. Biochem. J. 81, 24 P.

Mitchell, P. \& Moyle, J. (1956a). Osmotic function and structure in bacteria. Symp. Soc. gen. Microbiol. 6, 150.

Mitcheld, P. \& Moyle, J. (1956b). Liberation and osmotic properties of the protoplasts of Micrococcus lysodeikticus and Sarcina lutea. J. gen. Microbiol. 15, 512.

Mrtchell, P. \& Moyle, J. (1958a). Group-translocation: a consequence of enzymecatalysed group-transfer. Nature, Lond. 182, 372.

Mitchell, P. \& Moyle, J. (1958b). Enzyme catalysis and group-translocation. Proc. Roy. Phys. Soc. (Edinb.), 27, 61.

Perers, R. A. (1939). Proteins and cell-organisation. In Perspectives in Biochemistry, p. 36. Ed. J. Needham \& D. E. Green. Cambridge University Press.

Robertson, R. N. (1960). Ion transport and respiration. Biol. Rev. 35, 231.

Waddington, C. H. (1959). The biophysics of animal form. In Nere Biology, vol. 30, p. 112. Ed. M. L. Johnson, M. Abercrombie \& G. E. Fogg. London: Penguin Books. 